\title{
PRODUÇÃO DE SERAPILHEIRA E CICLAGEM DE NUTRIENTES NA CULTURA DO CAJUEIRO ANÃO PRECOCE ${ }^{1}$
}

\author{
Ismail Soares², Janayna de Albuquerque Queiroz ${ }^{3}$, Vitor Hugo de Oliveira ${ }^{4}$, Lindbergue Araújo \\ Crisóstomo ${ }^{4}$ e Teógenes Senna de Oliveira ${ }^{2}$
}

\begin{abstract}
RESUMO - O estudo teve por objetivos: (a) avaliar a produção de biomassa proveniente da copa do cajueiroanão precoce, clone CCP 76, com idades de 2, 3, 8 e 9 anos de implantação da cultura; (b) determinar a taxa de decomposição das folhas; e (c) quantificar a contribuição de nutrientes potencialmente disponíveis na biomassa para serem reciclados no solo. O trabalho foi conduzido no Campo Experimental do Curu, no Município de Paraipaba, CE, no período de dezembro de 2003 a janeiro de 2005. Os tratamentos constituíam de pomares com 2, 3, 8 e 9 anos de implantação, distribuídos em delineamentos inteiramente casualizados com oito repetições. Para a coleta da serapilheira, utilizaram-se coletores de $1 \mathrm{~m}^{2} \mathrm{com}$ malha de $1 \mathrm{~mm}^{2}$ colocados a $40 \mathrm{~cm}$ da superfície do solo. Após a coleta, o material foi separado em frações: folhas, galhos, inflorescência, pedúnculo e castanha, que após secadas em estufa foram pesadas. Na fração folhas, determinaram-se os teores de N, P, K, Ca, Mg, S, Na, Cu, Fe, Mn e Zn. De cada planta, coletaram-se folhas prestes a cair, para determinar a taxa de decomposição. Utilizaram-se $12 \mathrm{~g}$ desse material seco em estufa, o qual foi colocado em bolsas de náilon com 20 x $20 \mathrm{~cm}$ e malha de $2 \mathrm{~mm}$, as quais foram distribuídas na superfície do solo e coletadas após 112, 233 e 369 dias. Em cada coleta, o material foi seco em estufa, determinando-se as quantidades remanescentes de biomassa e de nutrientes (N, P, K, Ca, Mg, S, Na, Cu, Fe, Mn e Zn). As plantas com 8 e 9 anos de implantação depositaram maiores quantidades de serapilheira durante o período do experimento e apresentaram os maiores potenciais de reciclagem de nutrientes. Durante o período do experimento, observou-se, de modo geral, que o processo de decomposição foi rápido nos primeiros quatro meses e, posteriormente, manteve-se lento até o término das observações. No cajueiro, a maior parte dos nutrientes foi liberada nos primeiros quatro meses de decomposição das folhas.
\end{abstract}

Palavras-chaves: Biomassa, decomposição e Anacardium occidentale L.

\section{LITTER PRODUCTION AND NUTRIENT CYCLES IN THE YOUNG DWARF CASHEW CULTURE}

\begin{abstract}
This study aimed to (a) to evaluate the biomass production from the crown of the young dwarf cashew, clone CCP 76, at 2, 3, 8 and 9 years of culture implantation, (b) to determine the rate of leaf decomposition and (c) to quantify nutrient contribution potentially available in the biomass to be recycled into the soil. The research was conducted at the Experimental Field of Curu, in Paraipaba-CE, from December, 2003 to January, 2005. The treatments consisted of orchards with trees of 2, 3, 8 and 9 years of plantation, distributed in a completely randomized design with eight replications. Litter was collected using $1 \mathrm{~m}^{2}$ collectors with $1 \mathrm{~mm}^{2}$ mesh place $40 \mathrm{~cm}$ above the soil surface. After sampling, the material was sorted out into portions of leaves, branches, inflorescence, peduncle and nut, oven dried and weighed. In the leaf portion, the compositions of $\mathrm{N}, \mathrm{P}, \mathrm{K}, \mathrm{Ca}, \mathrm{Mg}, \mathrm{S}, \mathrm{Na}, \mathrm{Cu}, \mathrm{Fe}, \mathrm{Mn}$ and $\mathrm{Zn}$ were determined. Leaves ready to fall were collected from
\end{abstract}

\footnotetext{
${ }^{1}$ Recebido em 20.12.2006 e aceito para publicação em 18.12.2007.

${ }^{2}$ Departamento de Ciências do Solo, da Universidade Federal do Ceará, UFC/CCA/DCS. Fortaleza-CE. E-mail : <ismail@ufc.br> .

${ }^{3}$ Bióloga, M.Sc., Rua Sírios, 63, Barra do Ceará, 60330-380, Fortaleza-CE. E.mail: <janainack@yahoo.com.br>.

4Embrapa Agroindústria Tropical, Cx.P. 3761, 60511-110 Fortaleza-CE. E-mail: < vitorho@gmail.com>e <lindberg@cnpat.embrapa.br>.
} 
each plant to determine their decomposition rate and $12 \mathrm{~g}$ of this material was stove dried, and placed in $20 \mathrm{~cm} \times 20 \mathrm{~cm}$ nylon bags and $2 \mathrm{~mm}$ mesh which were distributed on the soil surface and collected after 112, 233 and 369 days. In each collection, the material was oven dried and the remaining nutrient biomass ( $N, P, K, C a, M g, S, N a, C u, F e, M n$ and $\mathrm{Zn}$ ) was determined. The 8-and 9-year-old plants deposited greater quantities of litter during the experimental period and showed greater potential for nutrient recycling. During the experimental period it was generally observed that the decomposition process was fast in the first four months, remaining slow until the end of the observation period. Most of the nutrients in the cashew tree were released in the first four months of leaf composition.

Keywords: Biomass, decomposition and Anacardium occidentale L.

\section{INTRODUÇÃO}

A ciclagem de nutrientes abrange as trocas de elementos minerais entre os seres vivos e o ambiente que os circunda, centrando-se nas relações entre a vegetação e o solo, por meio dela, obtêm-se informações sobre a distribuição de nutrientes no ecossistema, podendo-se inferir sobre os fluxos entre os diferentes compartimentos (JORDAN, 1995). A maior parte dos nutrientes absorvidos pelas plantas retorna ao solo pela queda dos componentes senescentes da parte aérea e sua posterior decomposição. Esses fragmentos orgânicos, ao caírem sobre o solo, formam uma camada denominada serapilheira, que compreende folhas, caules, frutos, flores, bem como restos de animais e material fecal. Uma vez depositada sobre o solo a serapilheira protege-o contra a erosão, além de tornar-se uma fonte potencial de energia para as espécies consumidoras do ecossistema, ao participarem da ciclagem de nutrientes (NETO et al., 2001). Há vários trabalhos na literatura que apontam as folhas como os principais materiais formadores da serapilheira. Estudando a deposição de serapilheira em uma área reflorestada com espécies arbóreas no município de Limoeiro-SP, Moreira \& Silva (2004) observaram que a produção anual média de serapilheira foi $6.636 \mathrm{~kg} \mathrm{ha}^{-1}$, onde a fração folhas contribuiu com a maior percentagem (80\%).

Vários fatores afetam a quantidade de resíduos que irão formar a serapilheira, entre eles destacamse o clima, o solo, as características genéticas da espécie, a idade e a densidade de plantas. A influência das condições climáticas na taxa de produção anual da serapilheira pode ser observada nos estudos de Andrade et al. (2000), os quais verificaram que nas áreas com Mimosa caesalpiniifolia as maiores taxas de produção ocorreram no período de menor temperatura e precipitação, os quais estimularam as plantas a aumentar a queda de material senescente. O teor e a quantidade de nutrientes na serapilheira podem variar, para uma mesma espécie, em função do sítio, das características da planta e das do próprio elemento. Nas frações da serapilheira de acácia-negra, Schumacher et al. (2003) observaram que o maior teor de $\mathrm{P}$ foi observado nas frações flores e frutos, atribuído ao fato desse nutriente ter a função de estimular o crescimento, apressar a maturação e ajudar a formação de sementes, a respiração e absorção iônica de outros elementos.

O agronegócio do caju constitui-se de elevada importância econômica e social para as regiões tropicais. No Brasil, terceiro maior produtor mundial de caju, a atividade concentra-se principalmente na região Nordeste, com 94\% da produção nacional, principalmente nas faixas litorâneas e de transição dos Estados do Ceará, Rio Grande do Norte e Piauí (PIMENTEL, 1996). No Nordeste brasileiro, o cajueiro é geralmente cultivado em solos de baixa fertilidade natural, tornando-se necessário o desenvolvimento de sistemas de uso do solo caracterizados por uma eficiente ciclagem de nutrientes. Estudos realizados por Richards (1992), na Austrália, comprovaram que o cajueiro pode representar uma fonte de bioenergia renovável, desde que manejado adequadamente. Segundo esse autor, do total da biomassa proveniente da copa cerca de 107 kg de N, 13 kg de P e 63 kg de K são potencialmente disponíveis para serem reciclados no solo, mostrando sua importância como fonte de nutrientes. Apesar disso, o sistema de colheita empregado na maioria dos pomares existentes no Brasil, com a retirada e/ou queima das folhas sob a copa das plantas remove grandes quantidades de nutrientes.

Os objetivos do trabalho foram: (a) avaliar a produção de biomassa proveniente da copa do cajueiro anão precoce, com 2, 3, 8 e 9 anos de implantação da 
cultura; (b) determinar a taxa de decomposição das folhas provenientes da copa do cajueiro anão precoce e (c) quantificar a contribuição de nutrientes potencialmente disponíveis na biomassa das folhas para serem reciclados no solo.

\section{MATERIAL E MÉTODOS}

O experimento foi conduzido no Campo Experimental do Curu pertencente à Embrapa Agroindústria Tropical, coordenadas geográficas de $3^{\circ} 28$ ' $47^{\prime}$ 'S de latitude e 3909'47' $W$ de longitude e altitude de 31m, no município de Paraipaba, Ceará. O clima da região é do tipo Aw, segundo Köppen, ou seja, tropical chuvoso, apresentando o máximo de chuvas no outono e período seco no inverno, e o solo classificado como Neossolo Quartzarênico (EMBRAPA, 1999).

Foram selecionados quatro pomares de cajueiro anão precoce (variedade botânica da espécie Anacardium occidentale L, de acordo com LIMA (1988)), clone CCP 76 com quatro diferentes idades de implantação (2, 3, 8 e 9 anos). De cada idade selecionaram-se oito plantas, perfazendo um total de trinta e duas unidades experimentais.

No pomar com 2 anos de implantação, estava sendo conduzido um experimento de doses de $\mathrm{N}$ e K, as plantas selecionadas faziam parte do tratamento D3 e recebiam $4000 \mathrm{~g}$ de uréia por quinzena. Em dezembro de 2003 as plantas receberam via fertirrigação, 50g N planta $^{-1}$ (aplicado em duas parcelas). Durante o ano de 2004 essas plantas receberam 575g N planta-1 (aplicado em duas parcelas por mês), a dose de $\mathrm{P}\left(250,20 \mathrm{~g} \mathrm{P}_{2} \mathrm{O}_{5}\right.$ planta $^{-1}$ ) foi aplicada em fevereiro. Durante o mês de janeiro de 2005 não foi aplicado nenhum adubo na área.

Na área com plantas de 3 anos de implantação, o experimento envolvia fontes e doses de $\mathrm{N}$ no cultivo do cajueiro, as plantas escolhidas faziam parte do tratamento 1 no qual o fertilizante usado como fonte de $\mathrm{N}$ era o sulfato de amônio. Durante o ano de 2004 as plantas receberam $120 \mathrm{~g} \mathrm{~N}$ planta $^{-1}$, $260 \mathrm{~g}$ $\mathrm{P}_{2} \mathrm{O}_{5}$ planta $^{-1}$ e 800g K $\mathrm{Oplanta}^{-1}$. O fertilizante usado como fonte de $\mathrm{P}$ (superfosfato simples) foi aplicado em dose única no mês de janeiro, enquanto que os fertilizantes usados como fonte de $\mathrm{N}$ e K(cloreto de potássio) foram aplicados, via fertirrigação, em seis parcelas com intervalo de 30 dias, nos meses de janeiro, fevereiro, março, abril, maio e junho. Em janeiro de 2005 as plantas receberam 260g $\mathrm{P}_{2} \mathrm{O}_{5}$ planta $^{-1}$ (aplicado em dose única), $20 \mathrm{~g} \mathrm{~N}_{\text {planta }}{ }^{-1}$ e $133 \mathrm{~g} \mathrm{~K}_{2} \mathrm{O}$ planta $^{-1}$.
No pomar com 8 anos de implantação estava sendo conduzido um experimento para avaliar o efeito do $\mathrm{N}$, $\mathrm{P}$ e K. As plantas selecionadas faziam parte do tratamento 16 , cada planta recebia anualmente $600 \mathrm{~g}$ de $\mathrm{N}$ e $450 \mathrm{~g}$ de $\mathrm{P}_{2} \mathrm{O}_{5}$. No ano de 2004 as plantas receberam $120 \mathrm{~g}$ $\mathrm{N}_{\text {planta }}{ }^{-1}$ e $450 \mathrm{~g} \mathrm{~K}_{2} \mathrm{O}$ planta $^{-1}$, via fertirrigação. Nesse ano não foi aplicado nenhum adubo contendo P. A aplicação dos fertilizantes contendo $\mathrm{N}$ e $\mathrm{K}$ foi semelhante à executada nas plantas com 3 anos.

Na área com plantas de 9 anos de implantação estava sendo conduzido um experimento para avaliar o comportamento de clones de cajueiro anão precoce sob diferentes regimes de irrigação. Em dezembro de 2003 as plantas receberam via fertirrigação, 23g $\mathrm{N}_{\text {planta }}{ }^{-1}$ e $15 \mathrm{~g} \mathrm{~K}_{2} \mathrm{O}$ planta $^{-1}$. No ano de 2004 as plantas receberam 212g N planta ${ }^{-1}$, 141g K $\mathrm{g}_{2}$ planta $^{-1}$ e $250 \mathrm{~g}$ $\mathrm{P}_{2} \mathrm{O}_{5}$ planta $^{-1}$.

Para estimar a quantidade de serapilheira produzida utilizou-se 1 coletor de $1 \mathrm{~m}^{2}$, sob a copa de cada planta, confeccionado em madeira e tela de náilon (malha de $1 \mathrm{~mm}$ ), suspensos a $40 \mathrm{~cm}$ do solo. A serapilheira interceptada por cada coletor foi coletada durante o período de Dezembro de 2003 a Janeiro de 2005, com intervalo de três meses. Posteriormente, foi limpa e separada em frações: folhas, galhos, inflorescência, pedúnculo e castanha. Após a separação, cada fração foi seca em estufa com circulação e renovação de ar a $65^{\circ} \mathrm{C}$, por 72 horas para determinar o peso seco.

Da fração folhas, foram retiradas amostras homogêneas, as quais foram trituradas em moinho do tipo Willey e posteriormente analisadas quanto aos teores de N, P, K, S, Ca, Mg, Na, Fe, Mn, Zn e Cu, de acordo com metodologia descrita por Silva (1999). $\mathrm{O} \mathrm{N}$ foi mineralizado por digestão sulfúrica e quantificado por destilação pelo método semimicro-Kjeldahl. Os demais nutrientes foram mineralizados em extrato nitro-perclórico, sendo o P determinado por espectrofotometria pelo método azul de molibdênio, o $\mathrm{S}$ por turbidimetria do cloreto de bário, o K e Na por fotometria de chama, e Ca, Mg, Fe, Zn, Mn e Cu por espectrometria de absorção atômica.

De cada planta, coletaram-se folhas prestes a cair para determinar a taxa de decomposição e o teor de nutrientes. As folhas foram secas em estufa com circulação e renovação de ar a $65^{\circ} \mathrm{C}$, durante 72 horas. Em seguida, pesaram-se $12 \mathrm{~g}$ de matéria seca o qual foi acondicionado em bolsas de decomposição de $400 \mathrm{~cm}^{2}(20$ x 20 cm)

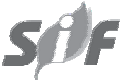

R. Árvore, Viçosa-MG, v.32, n.1, p.173-181, 2008 
confeccionadas com tela de náilon de malha $2 \mathrm{~mm}$. Em Janeiro de 2004 instalaram-se três bolsas na superfície do solo sob a copa de cada planta.

O processo de decomposição foi acompanhado de Janeiro de 2004 a Janeiro de 2005. As folhas em decomposição foram limpas com auxílio de um pincel e secas em estufa com circulação e renovação de ar a $65^{\circ} \mathrm{C}$ durante 72 horas. Posteriormente foi determinado o peso seco para o cálculo da constante de decomposição (k), segundo Olson (1963), citado por Aidar \& Joly (2003), pela fórmula $\mathrm{k}=$ - Ln [1 - (dPS/Pso)], onde dPS corresponde à perda de peso seco no período considerado e Pso ao peso inicial do período considerado.

As folhas em decomposição foram trituradas em moinho tipo Willey e mineralizadas para análise química, seguindo a metodologia descrita por Silva (1999).

O delineamento experimental utilizado foi inteiramente casualizado. As análises de variância e de regressão dos dados foram realizadas utilizando o programa SAEG (Sistema de Análise Estatística e Genética) e as médias foram comparadas pelo teste de Tukey com nível de 5\% de significância. Os coeficientes das equações de regressão foram analisados pelo teste t a 5\% de significância.

\section{RESULTADOS E DISCUSSÃO}

As plantas com 2, 3, 8 e 9 anos de idade produziram 4,46, 4,25, 8,54 e 7,88 $\mathrm{Mg} \mathrm{ha}^{-1}$ ano $^{-1}$ de serapilheira, respectivamente. No período de Dezembro de 2003 a Março de 2004 (coleta 1) as plantas com 2 anos de idade apresentaram menor produção de serapilheira, enquanto que as com 8 e 9 anos apresentaram maior produção de serapilheira; nas plantas com 3 anos observou-se produção intermediária. No período de Março a Junho de 2004 (coleta 2) as plantas com 8 anos de idade produziram maior quantidade de serapilheira, enquanto que as com 3 anos exibiram menor produção, nas plantas com 2 e 9 anos verificaram-se produções intermediárias (Figura 1). A queda de folhas em maior intensidade no período da coleta 2 coincide com o observado nos estudos de Mesquita (2003) relacionados à fenologia do cajueiro comum e anão. A este respeito, Frota (1988) afirmou que a queda de folhas do cajueiro ocorre durante todo o ano, aumentando acentuadamente a partir de Maio, logo após as precipitações pluviais mais intensas, com pico entre Junho e Agosto. No período de Junho a Setembro de 2004 (coleta 3) verificou-se que as plantas com 8 e
9 anos apresentaram maiores produções de serapilheira, enquanto que as plantas com 2 e 3 anos apresentaram menores produções. Pode-se atribuir a maior produção de serapilheira observada nas plantas mais velhas ao maior volume da copa, em relação às plantas mais jovens. A produção de serapilheira aumentou em todas as idades entre os períodos de coleta, exceto entre as coletas 1 e 2 para plantas com 9 anos, ocorrendo maior intensidade de deposição no período de Setembro de 2004 à Janeiro de 2005 (coleta 4) em relação aos anteriores, vista a coincidência com a fase de frutificação, a qual iniciase em Julho, estendendo-se até Fevereiro (BARROS et al., 1984).

O potencial de reciclagem de $\mathrm{N}, \mathrm{P}, \mathrm{Mg}, \mathrm{S}$ e $\mathrm{Na}$ foi maior nas plantas com 8 e 9 anos e foi menor nas de 2 e 3 anos de implantação (Tabela 1). O maior potencial de reciclagem de $\mathrm{N}$ nas plantas com 8 e 9 anos é atribuído a maior deposição de serapilheira (Figura 1). Costa et al. (2004) também constataram que os maiores potenciais de reciclagem de nutrientes na serapilheira de leguminosas arbóreas mostraram-se fortemente associados com a maior produção de fitomassa. Em média, o maior potencial de reciclagem de $\mathrm{N}$ observado no cajueiro em relação aos demais macronutrientes pode ter sido o resultado da aplicação de fertilizantes nitrogenados nas áreas do experimento. As plantas com 8 e 9 anos apresentaram os maiores potenciais de reciclagem de $\mathrm{P}, \mathrm{Mg}$, S e Na (Tabela 1), o qual pode ser atribuído a maior deposição de serapilheira por essas plantas (Figura 1).

Apesar dos teores de K, Ca, Cu e Mn na fração folha da serapilheira das plantas com 2 e 3 anos de idade serem superiores aos verificados nas plantas de 8 e 9 anos (Tabela 2), o potencial de reciclagem desses nutrientes não diferiu estatisticamente entre as plantas com diferentes idades (Tabela 1). Assim, o potencial de reciclagem de nutrientes no cajueiro em ordem decrescente foi: $\mathrm{N}>\mathrm{Ca}>\mathrm{Mg}>\mathrm{Na}>\mathrm{K}>\mathrm{S}>\mathrm{P}$ para os macronutrientes, e $\mathrm{Fe}>\mathrm{Mn}>\mathrm{Zn}>\mathrm{Cu}$ para os micronutrientes.

Em todas as plantas o processo de decomposição das folhas foi semelhante, com uma fase inicial rápida (cerca de 112 dias após a instalação das bolsas de serapilheira), seguida de outra mais lenta (Figura 2). Esse comportamento coincide com o observado por Aita e Giacomini (2003), em plantas de cobertura de solo, por Semwal et al. (2003) estudando espécies arbóreas e por Moretto e Distel (2003) trabalhando com Poa ligularis e Stipa gyneriodes. 


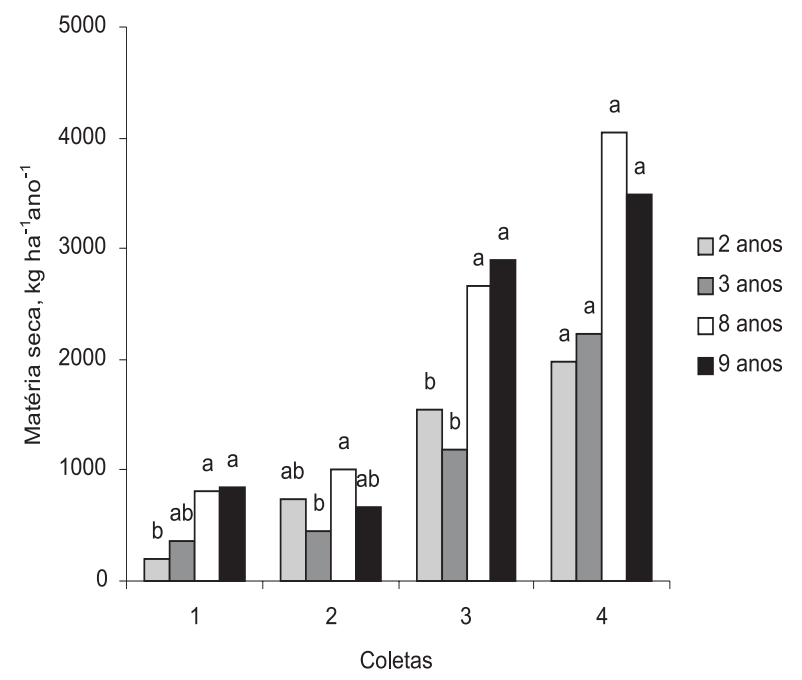

Médias seguidas de mesmas letras dentro de cada coleta não diferem entre si, pelo teste de Tukey a 5\% de probabilidade.

Figura 1 - Produção total de serapilheira do cajueiro-anão precoce, clone CCP 76, com diferentes idades de implantação durante o período de dezembro de 2003 à Janeiro 2005.

Figure 1 -Total young dwarf cashew litter production, clone CCP 76, at different plantation ages from December from 2003 to January of 2005.

As plantas com 8 anos de idade de implantação apresentaram os maiores valores de $\mathrm{k}$, indicando que nessa área a decomposição do material foi mais intensa (Figura 3), evidenciado pelo menor percentual de biomassa remanescente nas bolsas de serapilheira (Figura 2). Em média, o valor de k observado nas plantas com 8 anos foi de 0,65 , sendo inferior ao encontrado por Gama-Rodrigues et al. (2003) em espécies nativas de florestas. Estudando a decomposição da camada de serapilheira de Mimosa caesalpiniifolia, Andrade et al. (2000) obtiveram valor de $\mathrm{k}$ de 1,22, indicando que a decomposição da camada de serapilheira dessa espécie é mais rápida que a de cajueiro. A lenta decomposição da serapilheira do cajueiro também foi verificada nos estudos de Carvalho (2002), que atribuiu esse comportamento ao elevado teor de lignina no material formador da serapilheira. A taxa de decomposição da serapilheira na área com cajueiro de 8 anos também pode ser atribuída ao fato do material quando mantido sobre o solo, propiciando melhores condições de umidade e proliferação das comunidades de micro e macrorganismos. Sankaran (1993) encontrou correlação positiva entre a decomposição da serrapilheira e o teor de umidade na serrapilheira biomasssa de eucalipto (Eucalyptus tereticornis), teca (Tectona grandis) e albizia (Parasenianthes falcataria).

Observou-se um acúmulo de $\mathrm{N}$ nas folhas em decomposição provenientes das plantas com 2 e 3 anos de implantação ao longo do tempo (Tabelas 3, 4, 5 e 6). Esse comportamento também foi reportado nos estudos de Guo \& Sims (1999) e Gama-Rodrigues et al. (2003). Nas folhas provenientes das plantas com 8 anos constatou-se aumento na liberação do $\mathrm{N}$ ao longo do tempo, seguindo o mesmo comportamento observado para a biomassa remanescente de folhas nas bolsas de serapilheira (Figura 2). Resultados similares foram observados por Aita \& Giacomini (2003) e Moretto \& Distel (2003), estudando a decomposição e liberação de nutrientes na serapilheira de leguminosas e gramíneas, respectivamente.

Tabela 1 - Potencial de reciclagem anual de nutrientes das folhas da serapilheira do cajueiro-anão precoce, clone CCP 76, com diferentes idades de implantação

Table 1 - Annual recycling potential of nutrients in the leaves of the young dwarf cashew litter, clone CCP 76, at different plantation ages

\begin{tabular}{|c|c|c|c|c|c|c|c|c|c|c|c|}
\hline \multirow{2}{*}{$\begin{array}{l}\text { Idades } \\
\text { (anos) }\end{array}$} & $\mathrm{N}$ & $\mathrm{P}$ & $\mathrm{K}$ & $\mathrm{Ca}$ & $\mathrm{Mg}$ & $\mathrm{S}$ & $\mathrm{Na}$ & $\mathrm{Gu}$ & $\mathrm{Fe}$ & Mn & $\mathrm{Zn}$ \\
\hline & \multicolumn{7}{|c|}{$\mathrm{kg} \mathrm{ha}^{-1}$ ano $^{-1}$} & \multicolumn{4}{|c|}{$\mathrm{mg} \mathrm{ha}^{-1}$ ano $^{-1}$} \\
\hline 2 & 28,32 bc & $0,59 a b$ & 8,83a & $26,68 a$ & $9,56 a b$ & $1,74 a b$ & 7,91bc & $10,59 a$ & $370,11 b$ & $587,92 \mathrm{a}$ & $34,74 c$ \\
\hline 3 & $26,25 c$ & $0,50 b$ & $7,45 a$ & $26,88 a$ & $6,43 \mathrm{~b}$ & $1,72 b$ & $5,77 \mathrm{c}$ & $9,34 a$ & $376,45 b$ & $557,57 a$ & $40,41 \mathrm{bc}$ \\
\hline 8 & $57,31 \mathrm{a}$ & $0,97 a$ & $11,07 a$ & $30,02 \mathrm{a}$ & $13,90 a$ & $3,27 a$ & $13,72 a$ & $16,05 a$ & $806,91 \mathrm{a}$ & 559,83a & $70,35 a b$ \\
\hline 9 & $48,85 a b$ & $0,94 a$ & $10,74 a$ & $32,63 a$ & $14,48 a$ & $3,21 \mathrm{ab}$ & $12,43 a b$ & $15,02 \mathrm{a}$ & 714,08ab & $378,01 \mathrm{a}$ & $81,92 a$ \\
\hline Média & 40,18 & 0,75 & 9,52 & 29,05 & 11,09 & 2,49 & 9,96 & 12,75 & 566,89 & 520,83 & 56,85 \\
\hline CV(\%) & 38,58 & 35,77 & 51,45 & 34,85 & 37,27 & 44,18 & 40,12 & 39,46 & 43,96 & 42,10 & 41,84 \\
\hline
\end{tabular}

Médias seguidas de mesmas letras nas colunas não diferem entre si, pelo teste de Tukey a 5\% de probabilidade. 


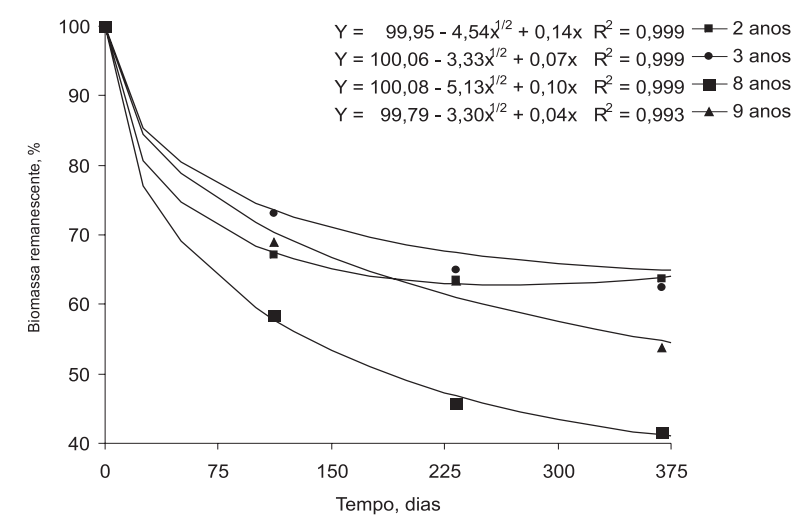

Figura 2 - Biomassa remanescente de folhas nas bolsas de serapilheira de plantas de cajueiro-anão precoce, clone CCP 76, com diferentes idades de implantação.

Figure 2-Residual leaf biomass in the litterbags of young dwarf cashew plants, clone CCP 76, at different plantation ages.

Em relação aos conteúdos iniciais de $\mathrm{P}$ e K nas folhas em decomposição (Tabela 3), observou-se em todas as plantas com diferentes idades a liberação desses nutrientes nos primeiros 112 dias (Tabela 4), sendo que nas plantas com 8 e 9 anos a liberação foi lenta e contínua de $\mathrm{P}$ após este período. A diminuição com o tempo do conteúdo de $\mathrm{P}$ também foi observada nos estudos de decomposição da palhada de milho realizados por Wisniewisk \& Holtz (1997). A grande liberação do Ké relacionada ao fato desse elemento não ser constituinte de nenhum composto orgânico existente na planta, e estar presente na forma iônica, o que facilita a sua liberação da célula após o rompimento da membrana plasmática (OLIVEIRA et al., 1999). De modo geral, verificou-se acúmulo de Ca nas folhas em decomposição. De acordo com Boeger et al. (2005) o acúmulo de Ca nos resíduos orgânicos em decomposição ocorre em função da sua baixa mobilidade, como também pode ser resultante da formação de cristais de oxalato de cálcio nos tecidos foliares.

Observou-se que nas folhas do cajueiro em decomposição, o Mg foi liberado em média de 57\% nos primeiros 112 dias (Tabela 4), em seguida, houve acúmulo desse nutriente. Nas plantas com 2 e 3 anos o $\mathrm{S}$ acumulou ao longo do tempo, enquanto que nas plantas com 8 e 9 anos observou-se acúmulo desse nutriente nos primeiros 112 dias (Tabela 4), em seguida, a liberação foi, em média de 93,28\% (Tabelas 5 e 6). $\mathrm{O}$ acúmulo de $\mathrm{S}$ nas plantas com 3 e 8 anos pode ser o resultado da aplicação de sulfato de amônio ao solo, enquanto que nas demais pode-se atribuir esse aumento à baixa mobilidade desse nutriente nos tecidos vegetais (MALAVOLTA, 1997). Em relação aos demais macronutrientes o $\mathrm{Na}$ foi o mais liberado nos primeiros 112 dias em todas as folhas em decomposição das plantas com diferentes idades (Tabela 4), isso pode ser explicado pela sua alta mobilidade na planta.
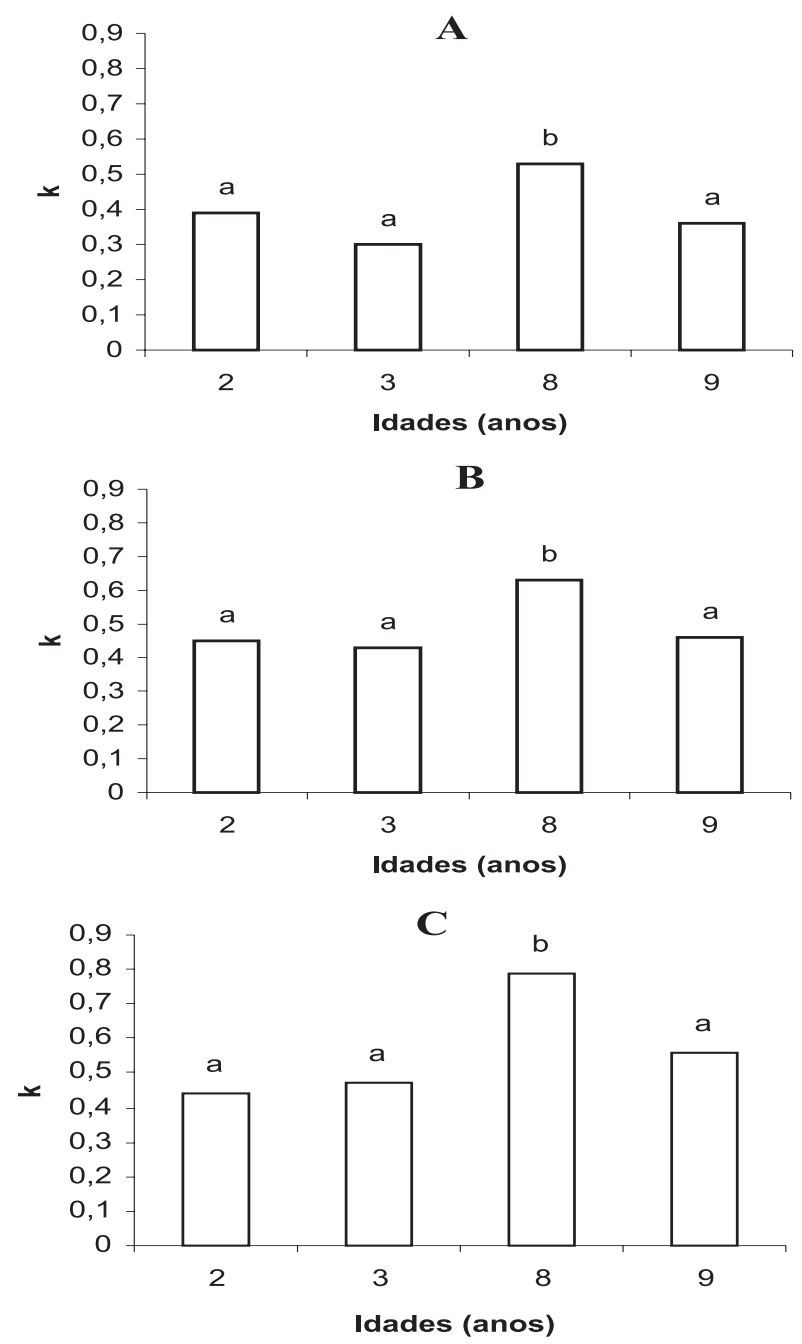

Figura 3 - Constante de decomposição (k) das folhas do cajueiro anão precoce, clone CCP 76, com diferentes idades de implantação aos 112 (A), 233 (B) e 369 (C) dias de instalação das bolsas de serapilheira na área experimental.

Figure 3 - Decomposition constant ( $k$ ) for the young dwarf cashew leaf, clone CCP 76, at different plantation ages on 112 (A), 233 (B) and 369 (C) days of litterbag installation in the experimental area. 
Tabela 2 - Teores de nutrientes nas folhas da serapilheira do cajueiro anão precoce, clone CCP 76, com diferentes idades de implantação

Table 2 - Nutrient contents in the leaves of the young dwarf cashew litter, clone CCP 76, at different plantation ages

\begin{tabular}{|c|c|c|c|c|c|c|c|c|c|c|c|}
\hline \multirow{2}{*}{$\begin{array}{l}\begin{array}{l}\text { Idades } \\
\text { (anos) }\end{array} \\
\end{array}$} & $\mathrm{N}$ & $\mathrm{P}$ & K & $\mathrm{Ca}$ & Mg & $\mathrm{S}$ & $\mathrm{Na}$ & $\mathrm{Gu}$ & $\mathrm{Fe}$ & Mn & $\mathrm{Zn}$ \\
\hline & & & & $\mathrm{g} \mathrm{kg}^{-1}$ & & & & \multicolumn{4}{|c|}{$\mathrm{mg} \mathrm{kg}^{-1}$} \\
\hline 2 & $9,32 a$ & $0,19 a$ & $2,74 a$ & $8,83 a$ & $3,11 \mathrm{a}$ & $0,56 a$ & $2,64 a$ & $3,51 a$ & $129,50 a$ & $191,04 a$ & $\overline{11,46 \mathrm{ab}}$ \\
\hline 3 & $8,50 \mathrm{bc}$ & $0,16 b$ & $2,38 a$ & $8,86 a$ & $2,09 b$ & $0,56 a$ & $1,87 b$ & $3,07 \mathrm{ab}$ & $121,35 a$ & $184,85 a$ & $13,44 a b$ \\
\hline 8 & $9,15 \mathrm{ab}$ & $0,15 b$ & $1,72 b$ & $4,81 b$ & $2,24 b$ & $0,52 \mathrm{a}$ & $2,21 a b$ & $2,60 \mathrm{bc}$ & $130,77 \mathrm{a}$ & $90,96 b$ & $11,36 b$ \\
\hline 9 & $8,19 c$ & $0,16 b$ & $1,73 \mathrm{~b}$ & $5,53 \mathrm{~b}$ & $2,50 \mathrm{~b}$ & $0,54 \mathrm{a}$ & $2,08 a b$ & $2,41 c$ & $116,55 a$ & $61,58 \mathrm{~b}$ & $13,66 \mathrm{a}$ \\
\hline Média & 8,79 & 0,17 & 2,14 & 7,01 & 2,49 & 0,55 & 2,20 & 2,90 & 124,54 & 132,11 & 12,48 \\
\hline $\mathrm{CV}(\%)$ & 6,29 & 12,41 & 19,80 & 14,75 & 13,12 & 22,39 & 18,85 & 12,76 & 22,98 & 20,24 & 13,06 \\
\hline
\end{tabular}

Médias seguidas de mesmas letras nas colunas não diferem entre si, pelo teste de Tukey a 5\% de probabilidade.

Tabela 3 - Conteúdo de nutrientes na folha do cajueiro-anão precoce, clone CCP 76, com diferentes idades após o plantio, antes da instalação do experimento

Table 3 - Nutrient content in the young dwarf cashew leaf, clone CCP 76, at different plantation ages, before the installation of the experiment

\begin{tabular}{|c|c|c|c|c|c|c|c|c|c|c|c|}
\hline \multirow{2}{*}{$\begin{array}{l}\text { Idades } \\
\text { (anos) } \\
\end{array}$} & $\mathrm{N}$ & $\mathrm{P}$ & $\mathrm{K}$ & $\mathrm{Ca}$ & $\mathrm{Mg}$ & $\mathrm{S}$ & $\mathrm{Na}$ & $\mathrm{Gu}$ & $\mathrm{Fe}$ & Mn & $\mathrm{Zn}$ \\
\hline & \multicolumn{7}{|c|}{$\mathrm{mg} \mathrm{bolsa}^{-1}$} & \multicolumn{4}{|c|}{$\mu \mathrm{g} \mathrm{bolsa}^{-1}$} \\
\hline 2 & $86,61 \mathrm{ab}$ & $8,63 b$ & $54,08 a$ & $106,14 a$ & $24,12 b$ & $4,87 c$ & $53,61 a$ & $26,20 a$ & $1577,21 \mathrm{a}$ & $1860,07 \mathrm{ab}$ & $72,14 a$ \\
\hline 3 & $76,94 b$ & $8,88 b$ & $49,57 a$ & $110,10 a$ & $23,81 b$ & $7,24 b$ & $62,58 a$ & $14,58 b$ & $1530,42 a$ & $2295,75 a$ & $70,62 a$ \\
\hline 8 & $92,89 a$ & 9,13ab & $64,79 a$ & $75,04 b$ & $33,62 \mathrm{a}$ & $8,78 a$ & $60,47 a$ & $12,60 b$ & $1570,52 a$ & $1501,63 b$ & 59,19a \\
\hline 9 & $80,75 a b$ & $10,04 a$ & $63,37 a$ & $77,45 b$ & $32,98 a$ & $7,08 \mathrm{~b}$ & $54,73 a$ & $15,96 b$ & $1181,91 a$ & $957,08 c$ & $78,97 a$ \\
\hline Média & 84,30 & 9,17 & 57,95 & 92,18 & 28,63 & 6,99 & 57,85 & 17,33 & 1465,01 & 1653,63 & 70,13 \\
\hline $\mathrm{CV}(\%)$ & 11,27 & 9,05 & 21,27 & 12,91 & 15,46 & 6,27 & 13,92 & 18,90 & 28,90 & 20,42 & 29,29 \\
\hline
\end{tabular}

Médias seguidas de mesmas letras nas colunas não diferem entre si, pelo teste de Tukey a 5\% de probabilidade.

Tabela 4 - Conteúdo de nutrientes remanescentes na folha do cajueiro-anão precoce, clone CCP 76, com diferentes idades após o plantio, 112 dias após a instalação do experimento

Table 4 - Residual nutrient content in the young dwarf cashew leaf, clone CCP 76, at different plantation ages, 112 days after the installation of the experiment

\begin{tabular}{|c|c|c|c|c|c|c|c|c|c|c|c|}
\hline \multirow{2}{*}{$\begin{array}{l}\text { Idades } \\
\text { (anos) } \\
\end{array}$} & $\mathrm{N}$ & $\mathrm{P}$ & $\mathrm{K}$ & $\mathrm{Ca}$ & $\mathrm{Mg}$ & $\mathrm{S}$ & $\mathrm{Na}$ & $\mathrm{Gu}$ & $\mathrm{Fe}$ & Mn & $\mathrm{Zn}$ \\
\hline & \multicolumn{7}{|c|}{$\mathrm{mg} \mathrm{bolsa}^{-1}$} & \multicolumn{4}{|c|}{$\mu$ bolsa $^{-1}$} \\
\hline 2 & $94,38 a$ & 1,86ab & $5,24 b$ & $140,98 a$ & $15,24 a b$ & $10,46 b$ & $2,80 a$ & $36,29 b$ & $3405,01 a$ & 1013,62ab & $154,41 c$ \\
\hline 3 & $91,70 \mathrm{a}$ & 1,87ab & $10,71 a$ & $136,03 a$ & $11,08 b$ & $15,75 a$ & $3,95 a$ & $35,24 b$ & $3725,53 a$ & $1152,00 a$ & $212,14 b$ \\
\hline 8 & $87,20 a$ & $1,54 b$ & $6,01 b$ & $82,57 \mathrm{c}$ & $19,41 \mathrm{a}$ & $9,47 b$ & 3,73a & $35,90 b$ & $2644,78 a$ & 831,12 bc & $165,25 b c$ \\
\hline 9 & $80,26 a$ & $2,37 a$ & 7,89ab & $111,03 b$ & $19,27 a$ & $9,15 b$ & $3,92 a$ & 53,83a & $3592,94 a$ & $752,42 c$ & $291,02 a$ \\
\hline Média & 88,39 & 1,91 & 7,46 & 117,65 & 16,25 & 11,21 & 3,60 & 40,32 & 3342,07 & 937,29 & 205,70 \\
\hline CV(\%) & 17,12 & 23,14 & 28,03 & 15,07 & 28,68 & 23,41 & 31,99 & 30,93 & 29,82 & 14,59 & 17,43 \\
\hline
\end{tabular}

Médias seguidas de mesmas letras nas colunas não diferem entre si, pelo teste de Tukey a 5\% de probabilidade.

Em todas as folhas do cajueiro em decomposição observou-se acúmulo de $\mathrm{Cu}$, Fe e $\mathrm{Zn}$ nos primeiros 112 dias (Tabela 4), sendo que o acúmulo de Cu e Fe manteve-se ao longo do período estudado. Verificouse, em média, que a liberação de Zn foi de $18 \%$ no período de 233 a 369 dias após a instalação das bolsas (Tabela 6 ), enquanto que ocorreu liberação de 57\% de Mn nos primeiros 112 dias (Tabela 4) e posteriormente esse elemento acumulou nas folhas em decomposição (Tabelas 5 e 6). 
Tabela 5 - Conteúdo de nutrientes remanescentes na folha do cajueiro-anão precoce clone CCP 76, com diferentes idades após o plantio, 233 dias após a instalação do experimento

Table 5 - Residual nutrient content in the young dwarf cashew leaf, clone CCP 76, at different plantation ages, 233 days after installation of the experiment

\begin{tabular}{|c|c|c|c|c|c|c|c|c|c|c|c|}
\hline \multirow{2}{*}{$\begin{array}{l}\text { Idades } \\
\text { (anos) } \\
\end{array}$} & $\mathrm{N}$ & $\mathrm{P}$ & $\mathrm{K}$ & $\mathrm{Ca}$ & $\mathrm{Mg}$ & $S$ & $\mathrm{Na}$ & $\mathrm{Gu}$ & $\mathrm{Fe}$ & $\mathrm{Mn}$ & $\mathrm{Zn}$ \\
\hline & \multicolumn{7}{|c|}{$\mathrm{mg} \mathrm{bolsa}^{-1}$} & \multicolumn{4}{|c|}{$\mu \mathrm{g} \mathrm{bolsa}^{-1}$} \\
\hline 2 & $122,76 a$ & $2,08 a$ & $20,37 b$ & $121,20 a$ & $28,65 a$ & $5,54 b$ & $25,22 \mathrm{ab}$ & $25,88 b$ & $8018,43 a$ & $2222,22 a$ & $291,18 a$ \\
\hline 3 & 99,02ab & $2,04 a$ & $38,85 a$ & $89,55 b$ & $22,36 a b$ & $10,23 a$ & $28,33 a$ & $28,01 b$ & $6214,76 a b$ & 2128,93a & $298,88 a$ \\
\hline 8 & $78,64 b$ & $1,41 b$ & $17,04 b$ & $49,57 \mathrm{c}$ & $20,96 b$ & $4,70 \mathrm{~b}$ & $16,37 b$ & $29,15 b$ & 6423,53ab & $1071,85 b$ & $324,86 a$ \\
\hline 9 & $82,78 b$ & $2,20 \mathrm{a}$ & 27,53ab & $65,54 c$ & $27,97 a b$ & $6,09 \mathrm{~b}$ & $16,63 b$ & $42,66 a$ & $4471,95 b$ & 793,77b & $360,73 a$ \\
\hline Média & 95,80 & 1,93 & 25,95 & 81,47 & 24,98 & 6,64 & 21,64 & 31,42 & 6282,17 & 1554,20 & 318,91 \\
\hline CV(\%) & 18,68 & 17,61 & 32,91 & 20,06 & 21,09 & 28,55 & 31,77 & 28,81 & 25,69 & 27,14 & 35,87 \\
\hline
\end{tabular}

Médias seguidas de mesmas letras nas colunas não diferem entre si, pelo teste de Tukey a 5\% de probabilidade.

Tabela 6 - Conteúdo de nutrientes na folha do cajueiro-anão precoce clone CCP 76, com diferentes idades após o plantio, 369 dias após a instalação do experimento

Table 6 - Residual nutrient content in the young dwarf cashew leaf, clone CCP 76, at different plantation ages, 369 days after installation of the experiment

\begin{tabular}{|c|c|c|c|c|c|c|c|c|c|c|c|}
\hline \multirow{2}{*}{$\begin{array}{l}\text { Idades } \\
\text { (anos) } \\
\end{array}$} & $\mathrm{N}$ & $\mathrm{P}$ & K & $\mathrm{Ca}$ & Mg & $\mathrm{S}$ & $\mathrm{Na}$ & $\mathrm{Gu}$ & $\mathrm{Fe}$ & Mn & $\mathrm{Zn}$ \\
\hline & \multicolumn{7}{|c|}{$\mathrm{mg} \mathrm{bolsa}^{-1}$} & \multicolumn{4}{|c|}{$\mu$ bolsa $^{-1}$} \\
\hline 2 & $107,06 a$ & $2,06 a$ & $22,98 a$ & $105,69 a$ & $28,96 a$ & $5,94 b$ & $23,42 a$ & 47,94ab & $6740,51 \mathrm{ab}$ & $2094,26 a$ & $47,94 \mathrm{ab}$ \\
\hline 3 & $97,68 a$ & $2,32 a$ & $23,13 a$ & 93,83ab & $30,72 \mathrm{a}$ & $10,22 \mathrm{a}$ & $25,79 a$ & $66,05 a b$ & 7035,89a & $1691,04 a$ & $66,05 \mathrm{ab}$ \\
\hline 8 & $63,42 b$ & $1,11 b$ & $6,18 b$ & $43,22 c$ & $18,05 b$ & $4,23 b$ & $8,48 b$ & $44,09 b$ & $4774,23 \mathrm{bc}$ & $861,10 b$ & $44,09 b$ \\
\hline 9 & $70,64 b$ & 1,80ab & $16,50 a$ & 68,83bc & $27,00 \mathrm{a}$ & $5,29 b$ & $12,34 b$ & 68,96a & $4665,54 \mathrm{c}$ & $797,58 b$ & $68,96 a$ \\
\hline Média & 84,70 & 1,82 & 17,20 & 77,89 & 26,18 & 6,42 & 17,51 & 56,76 & 5804,04 & 1361,00 & 56,76 \\
\hline $\mathrm{CV}(\%)$ & 21,54 & 35,73 & 33,28 & 26,34 & 15,64 & 37,11 & 20,62 & 29,15 & 25,29 & 25,87 & 36,14 \\
\hline
\end{tabular}

Médias seguidas de mesmas letras nas colunas não diferem entre si, pelo teste de Tukey a 5\% de probabilidade.

\section{CONCLUSÃO}

As plantas com 8 e 9 anos de idade produziram maiores quantidades de serapilheira, em decorrência do maior volume da copa, assim como também apresentaram os maiores potenciais de reciclagem de nutrientes;

A fração folhas foi determinante na composição da serapilheira, com média de $81 \%$ de participação;

No cajueiro a maior parte dos nutrientes foi liberada nos primeiros quatro meses de decomposição das folhas, visto que nesse período a taxa de decomposição foi mais rápida.

\section{REFERÊNCIAS BIBLIOGRÁFICAS}

AIDAR, M.P.M.; JOLY, C.A. Dinâmica da produção e decomposição da serapilheira do araribá (Centrolobium tomentosum Guill. ex Benth.-Fabaceae) em uma mata ciliar, Rio JacaréPepira, São Paulo. Revista Brasileira de Botânica, v. 26, n.2, p. 193-202, 2003.
AITA, C.; GIACOMINI, S.J. Decomposição e liberação de nitrogênio de resíduos culturais de plantas de cobertura de solo solteiras e consorciadas. Revista Brasileira de Ciência do Solo, v.27, n.4, p.601-612, 2003.

ANDRADE, A.G.; COSTA, G.S.; FARIA, S.M. Deposição e decomposição da serapilheira em povoamentos de Mimosa caesalpiniifolia, Acacia mangium e Acacia holosericea com quatro anos de idade em planossolo. Revista Brasileira de Ciência do Solo, v. 24, n.4, p. 777-785, 2000.

BARROS, L.M. et al. A cultura do cajueiro anão. Fortaleza: Empresa de Pesquisa Agropecuária do Ceará-EPACE, 1984. 67p.

BOEGER, M.R.T.; WISNIEWSKI, C.; REISSMANN, C.B. Nutrientes foliares de espécies arbóreas de três estádios sucessionais de floresta ombrófila densa no sul do Brasil. Acta Botânica Brasilica, v. 19, n.1, p. 167-181, 2005. 
CARVAlHO, J.F.O. Dinâmica da matéria orgânica e de nutrientes em solo incorporado com diferentes resíduos vegetais. 2002. 36f. Dissertação (Mestrado em Solos e Nutrição de Plantas) - Universidade Federal do Ceará, Fortaleza, 2002.

COSTA, G.S.; FRANCO, A.A.; DAMASCENO, R.M. Aporte de nutrientes pela serapilheira em uma área degradada e revegetada com leguminosas arbóreas. Revista Brasileira de Ciência do Solo, v.28, n.5, p.919-927, 2004.

EMPRESABRASILEIRADE PESQUISA-EMBRAPA. Centro Nacional de Pesquisa de Solos. Sistema de classificação de solos. 2 ed. Rio de Janeiro: EMBRAPA, 1999. 411p.

FROTA, P.C.E. Clima e fenologia. In: LIMA, V.P.M.S. A cultura do cajueiro no Nordeste do Brasil. Fortaleza: BNB - ETENE, 1988. p.65-79.

GAMA-RODRIGUES; BARROS, N.F.; SANTOS, M.L. Decomposição e liberação de nutrientes do folhedo de espécies florestais nativas em plantios puros e mistos no sudeste da Bahia. Revista Brasileira de Ciência do Solo, v.27, n.6, p.1021-1031, 2003.

GOU, L.B.; SIMS, R.E.H. Litter decomposition and nutrient release via litter decomposition in New Zealand eucalypt short rotation forests.

Agriculture, Ecosystems and Enviroment, v.75, n.1, p.133-140, 1999.

JORDAN, C.F. Ciclagem de nutrientes e silvicultura de plantações na Bacia Amazônica. In: REUNIÃO BRASILEIRADE FERTILIDADE DO SOLO, 16.,1995, Ilhéus. Anais... Ilhéus, 1995. p.187-202.

LIMA, V.P.M.S. Botânica. In: LIMA, V.P.M.S. A cultura do cajueiro no Nordeste do Brasil. Fortaleza: BNB/ETENE, 1988. p.15-61.

MALAVOLTA, E.; VITTI, G.C.; OLIVEIRA, S.A. Avaliação do estado nutricional das plantas: princípios e aplicações. 2. ed. Piracicaba: POTAFOS, 1997. 319p.

MESQUITA, R.C.M. Fenologia e produtividade de progênies sexuada e assexuada de cajueiro comum e anão (Anacardium occidentale L.) sob irrigação. 2003. 89p. Tese (Doutorado em Fitotecnia) Universidade Federal do Ceará, Fortaleza, 2003.
MORETTO, A.S.; DISTEL, R.A. Decomposition of and nutrient dynamics in leaf litter and roots os Poa ligularis and Stipa gyneriodes. Journal of Arid Enviroments, v.55, n.3, p.503-514, 2003.

MOREIRA, P.R.; SILVA, O. A produção de serapilheira em área reflorestada. Revista Árvore, v.28, n.1, p.49-59, 2004.

NETO, T.A.C. et al. Deposição de serapilheira e mesofauna edáfica em áreas de eucalipto e Floresta secundária. Floresta e Ambiente, v.8, n.1, p.70-75, 2001.

OLIVEIRA, M.W. et al. Decomposição e liberação de nutrientes da palhada de cana-de-açúcar em campo. Pesquisa Agropecuária

Brasileira, v.34, n.12, p.2359-2362, 1999.

PIMENTEL, C.R.M. Impacto dos investimentos em pesquisa realizado pela EMBRAPA: o caso do cajueiro anão precoce. Fortaleza: EMBRAPA-CNPAT, 1996.15p.

RICHARDS, N.K. Cashew tree nutrition related to biomass accumulation, nutrient cycling in sandy red earths of Northern Territory, Australia. Scientia Horticulture, v.52, n.1-2, p.125-142, 1992.

SANKARAN, K.V. Decomposition of leaf litter of albizia (Paraserianthes falcataria), eucalypt (Eucalyptus tereticornis) and teak (Tectona grandis) in Kerala, India. Forest Ecology and Management, v.56, n.1-4, p.225-242, 1993.

SCHUMACHER, M.V. et al. Retorno de nutrientes via deposição de serapilheira em um povoamento de acácia-negra (Acacia mearnsii De Wild.) no Estado do Rio Grande do Sul. Revista Árvore, v.27, n.6, p.791-798, 2003.

SEMWAL, R.L. et al. Leaf litter decomposition and nutrient release patterns of six multipurpose tree species of central Himalaya, India. Biomass and Bioenergy, v.24, n.1, p.3-11, 2003.

SILVA, F.C. Manual de análise química de solos, plantas e fertilizantes. Brasília: EMBRAPA, 1999. 370p.

WISNIEWSKI, C.; HOLTZ, G.P. Decomposição da palhada e liberação de nitrogênio e fósforo numa rotação aveia-soja sob plantio direto. Pesquisa Agropecuária Brasileira, v. 32, n.11, p.1345-1350, 1997.

R. Árvore, Viçosa-MG, v.32, n.1, p.173-181, 2008 